\section{Fractional Anisotropy}

\author{
Nathan D. Zasler ${ }^{1}$ and Paul E. Kaplan ${ }^{2}$ \\ ${ }^{1}$ Concussion Care Centre of Virginia, Ltd, \\ Richmond, VA, USA \\ ${ }^{2}$ Capitol Clinical Neuroscience, Folsom, CA, \\ USA
}

\section{Definition}

Fractional anisotropy (FA) is a method that is used to emphasize and evaluate white matter fiber tracts. This method has been applied to MRI scans in diffusion weighted images. FA is a scalar value between 0 and 1 that describes the degree of anisotropy of a particular diffusion process. A value of 0 indicates that diffusion is isotropic, that is it is unrestricted or equally restricted in all directions. A value of 1 means that diffusion occurs only along one axis and is fully restricted along all other directions. FA is a measure often used in diffusion imaging where it is thought to reflect fiber density, axonal diameter, and myelination in white matter tracts. This type of nomenclature has also been applied to "fractional" procedures in molecular biology. Molecules can actually spread throughout the medium related to their thermal energy. The directional part of this spread is called anisotropy which literally defines the property of being directionally dependent indicating different properties in different directions.

\section{Current Knowledge}

The principal application of FA is in the imaging of white matter where the location, orientation, and anisotropy of the tracts can be measured. The architecture of the axons in parallel bundles, and their myelin sheaths, facilitate the diffusion of the water molecules preferentially along their main direction. Such preferentially oriented diffusion is called anisotropic diffusion.

\section{Cross-References}

$>$ DTI
$>$ DWI
$>$ MRI

\section{References and Readings}

Moseley, M. E., Liu, C., Rodriguez, S., et al. (2009). Advances in magnetic resonance neuroimaging. Neurologic Clinics, 27(1), 1-13.

O’Donnell, L. J., Kubicki, M., Shenton, M. E., Dreusicke, M. H., Grimson, W. E., \& Westin, C. F. (2006). A method for clustering white matter fiber tracts. American Journal of Neuroradiology, 27, 1032-1036.

Parker, G. J. M. (2004). Analysis of MR diffusion weighted images. The British Journal of Radiology, 77, S176-S185.

Shenton, M. E., Hamoda, H. M., Schneiderman, J. S., et al. (2012). A review of magnetic resonance imaging and diffusion tensor imaging findings in mild traumatic brain injury. Brain Imaging and Behavior, 6(2), 137-192. 